\title{
A JAPANESE CASE WITH AN UNUSUAL VARIANT OF CHROMOSOME 16 WITH AN EXTRA C-NEGATIVE, G-DARK SEGMENT IN THE SHORT ARM
}

\author{
Tadao ARINAMI ${ }^{1}$ and Ikuko KonDo ${ }^{2}$ \\ ${ }^{1}$ Ibaraki Prefectural Colony Hospital, Uchihara, Ibaraki 319-03, Japan \\ ${ }^{2}$ Department of Human Genetics, University of Tsukuba, \\ Tsukuba, Ibaraki 305
}

\begin{abstract}
Summary An unusual chromosome 16 is described in a family. The variant chromosome 16 had an additional C-band negative, G-band dark segment in the proximal region of the short arm. A proband was slightly retarded and had several minor anomalies. However, her mother and her maternal grandfather with the same variant chromosome 16 had no apparent phenotypic abnormalities. Thus, its absence of any consistent phenotypic effects in the family indicates that this is a variant chromosome, but its extra band differs from constituting heterochromatin in staining properties. The awareness of chromosomal heteromorphism is very important for clinical cytogenetics, because it may prevent a diagnostic misinterpretation.
\end{abstract}

\section{INTRODUCTION}

The chromosome 16 in the human karyotype has a well known site of remarkable polymorphic variation, usually involving changes in the site of heterochromatic C-band positive material. Recently, however, Thompson and Roberts (1987) reported an unusual variant of chromosome 16 with an extra G-dark band proximal to the centromere in the short arm which did not appear to involve standard C-band materials.

This report describes a similar variant in a family ascertained through a phenotypically abnormal child.

\section{MATERIALS AND METHODS}

A four month-old female was referred for karyotyping because of her mildly

Received February 8, 1988; revised version received March 10, 1988; Accepted March 12, 1988 
dysmorphic features including frontal bossing, flat occiput, depressed nasal bridge, large protruded ears and micrognathia. She was the $2,370 \mathrm{~g}$ product of a term pregnancy and had an asphyxia at birth. No remarkable family history was noted except for one spontaneous abortion of the maternal grandmother of the proband (Fig. 1). Her developmental milestones were moderately retarded: head control at

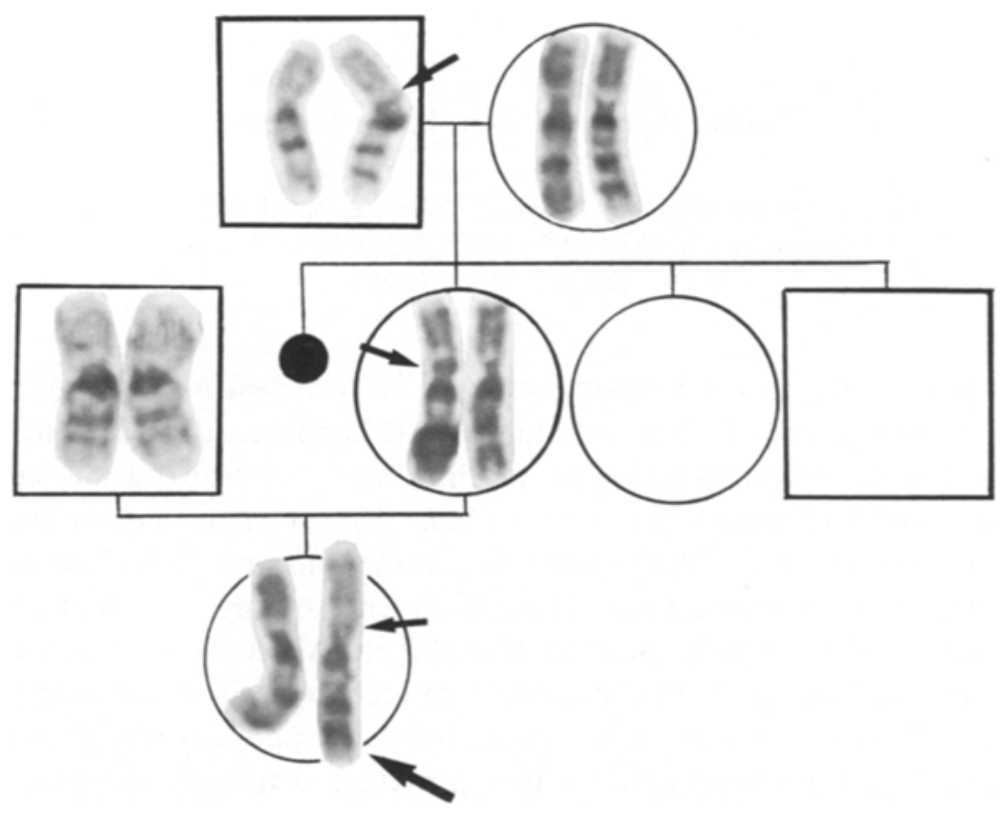

Fig. 1. Family pedigree and G-banded chromosome 16 pair from the proband, her parents and her maternal grandparents. The proband is indicated by a large arrow and the extra segment of chromosome 16 is indicated by a small arrow.

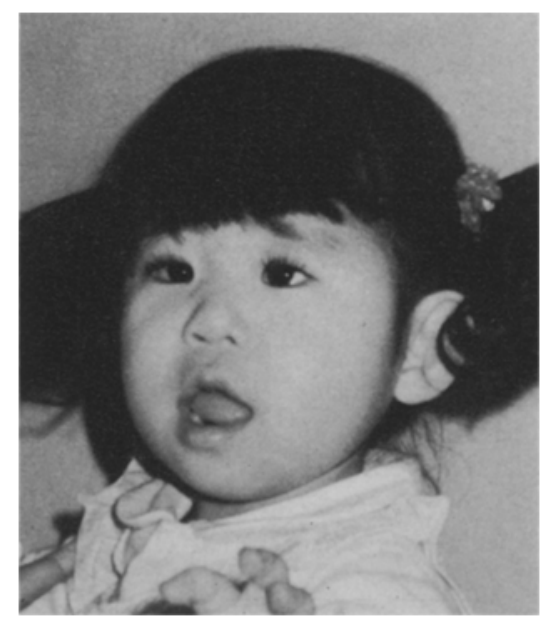

Fig. 2. Facial appearance of the proband at two years of age. 
four months of age, stand and walk without support at 18 and 21 months of age, respectively.

At two years of age, she had a short stature. Her height was $74.5 \mathrm{~cm}(-3$ S.D.). Her weight was $8.0 \mathrm{~kg}(-2.7$ S.D.). The chest and head circumferences were $44 \mathrm{~cm}$ and $45 \mathrm{~cm}(-1.5$ S.D.), respectively. The dysmorphic features marked at four months of age were also defined (Fig. 2).

The proband, her parents and the maternal grandparents were karyotyped using GTG and CBG procedures according to methods of Seabright (1971) and Sumner (1972), respectively.

\section{RESULTS AND DISCUSSION}

Cytogenetic investigation with GTG-banding in the proband showed a $16+$ with an extra chromosomal segment placed in the band p11 indicated by a small arrow in Fig. 1. The extra material did not stain with CBG-banding, indicating that it did not consist of the constitutive heterochromatin demonstrable by this technique (Fig. 3). Family study showed the same anomaly of chromosome 16 in the phenotypically normal mother and maternal grandfather (Fig. 1) and a deleted segment in other chromosomes was not detected in these subjects using high resolution banding technique (Ikeuchi, 1984) (data not shown). Therefore, it does not seem a derivative chromosome 16 from translocation chromosomes between the chromosome 16 and another chromosome. Though the karyotypic association with phenotypic abnormality of the proband cannot be completely excluded, the unusual chromosome 16 described in this report is more likely to be a variation because of its presence in phenotypically normal family members.

Recently, Thompson and Roberts (1987) reported a similar variant chromosome 16 in three families. The probands of these families had minor anomalies. However, the other family members with the same variant chromosome 16 had no phenotypical effects. The similar variant chromosome 16 was found in other Japanese families without common phenotypic effects (Oohashi et al., 1988). Therefore, this type of variant chromosome 16 appears to be present in different ethnic and racial

Fig. 3. C-banded chromosome 16 pair from the grandfather (GF), the mother (M) and the proband $(\mathrm{P})$.

Vol. 33, No. 3, 1988 
groups. In addition, a similar variant was also detected in chromosome 9 (Buckton et al., 1980; Archidiacono et al., 1984; Spedicato et al., 1985).

The origin and nature of this extra chromosomal materials, which apparently does not contain active genes but differs from usual heterochromatin in staining properties, remains to be established. At present, however, the awareness of this heteromorphism is very important for clinical cytogenetics because it may prevent a diagnostic misinterpretation.

\section{REFERENCES}

Archidiacono, N., Pecile, V., Rocchi, M., Dalpra, L., Nocera, G., and Simoni, G. 1984. A rare non-heterochromatic 9p + variant in two amniotic fluid cell cultures. Prenat. Diagn. 2: 231233

Buckton, K.E., O'Riodan, M.L., Ratcliffe, S., Slight, J., Mitchell, M., McBeath, S., Keay, A.J., Barr, D., and Short, M. 1980. A G-band study of chromosomes in liveborn infants. Ann. Hum. Genet. 23: 227-239

Ikeuchi, T. 1984. Inhibitory effect of ethidium bromide on mitotic chromosome concentration and its application to high resolution chromosome banding. Cytogenet. Cell Genet. 38: 56-61

Oohashi, T., Yokoyama, Y., Ishihara, Y., and Gunji, T. 1988. Cytogenetic features in a new variant of chromosome 16. 32nd Annual Meeting of the Japan Society of Human Genetics. Jpn. J. Human Genet. 33: 218

Seabright, M. 1971. A rapid banding technique for human chromosomes. Lancet ii, 971-972

Spedicato, F.S., DiComite, A., and Tohidast-Akrad, M. 1985. An unusual variant chromosome 9 with an extra C-negative, G-dark segment in the short arm. Clin. Genet. 28: 162-165

Sumner, A.T. 1972. A simple technique for demonstrating centromeric heterochromatin. Exp. Cell Res. 75: 304-306

Thompson, P.W., and Roberts, S.H. 1987. A new variant of chromosome 16. Hum. Genet. 76: $100-101$ 